Adapted from "Adopting Citizen

Observations in Operational Weather Prediction," by Thomas $\mathbf{N}$. Nipen (Norwegian Meteorological Institute), Ivar A. Seierstad, Christian Lussana, Jørn Kristiansen, and Øystein Hov. Published in BAMS online, February 2020. For the full citable article see DOI:10.1175/BAMS-D-18-0237.1.

\section{CITIZEN OBSERVATIONS FOR WEATHER
FORECASTS FOR WEATHER
FORECASTS}

t has long been argued that real-time citizen weather observations could significantly improve operational weather forecasting, particularly on small scales, by using them in the initialization of weather models. For example, pressure measurements from smartphones, due to their high network density, could revolutionize weather forecasting by improving the initialization of weather models, which in turn would produce forecasts that more accurately resolve frontal and convective weather systems.

Weather observations are also important in postprocessing NWP output, which helps forecasts better match the locally observed weather. The Norwegian Meteorological Institute (MET Norway) uses a state-of-the-art NWP model at $2.5-\mathrm{km}$ grid resolution and has traditionally adjusted the modeled temperatures on the fly using observations from roughly 200 professional weather stations nationally, owned and operated largely by MET Norway. Despite this, temperature forecast errors of $10^{\circ} \mathrm{C}$ are not unheard of in wintertime inversion conditions in areas where the observation network is sparse and adjustments are not possible. MET Norway and many other forecast providers would benefit from a denser network of stations to patch the holes that exist in the postprocessed field.

The demand for high-quality localized forecasts has risen with the use of and experience gained with our weather service, Yr ( $w w w$ .yr.no), which has as many as 10 million unique users worldwide per week. Yr is developed and operated jointly by MET Norway and the Norwegian Broadcasting Corporation. Feedback from users has provided valuable support and direction for R\&D on postprocessing. In response, MET Norway introduced observations from Netatmo, a manufacturer of professional weather stations, in operational temperature forecasts in March 2018 for locations in Norway, Denmark, 
(a) Observations

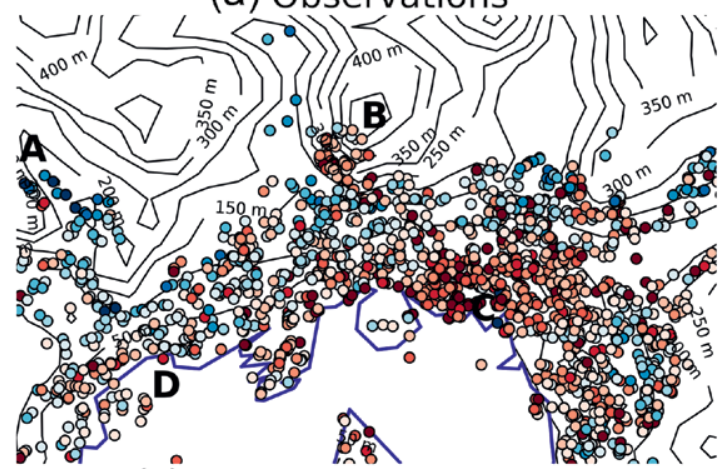

(c) Observations with $\mathrm{QC}$

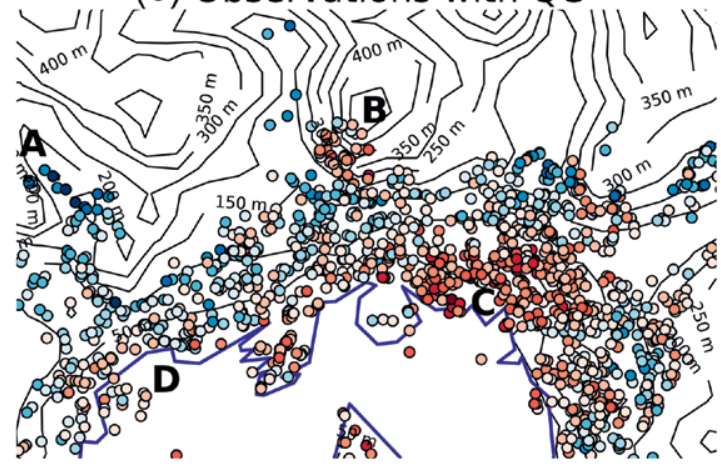

(b) NWP

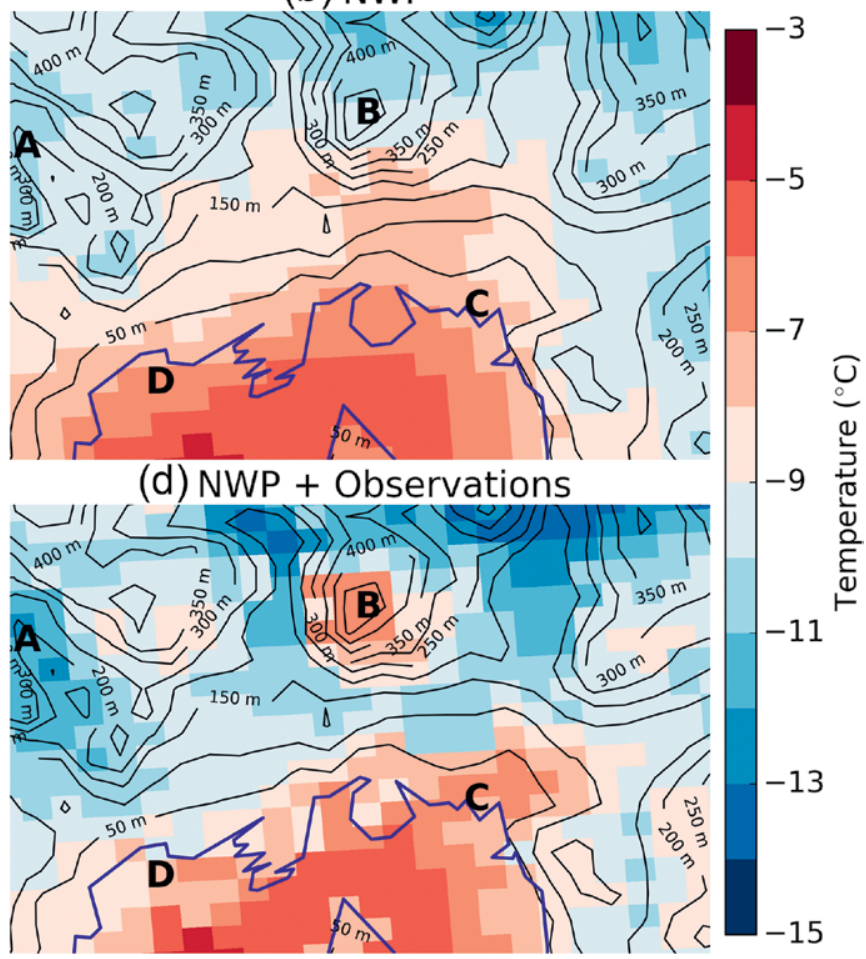

$\triangle$ 2-m temperatures over Oslo at 0500 UTC 28 Mar 2018 showing (a) all available observations from Netatmo; (b) model output from MEPS downscaled to $1 \mathrm{~km}$; (c) observations from (a) after the quality control procedures have been applied; and (d) postprocessed forecast incorporating model output from (b) and observations from (c). All panels use the colorbar on the right. Black lines show elevation contour lines with $50-\mathrm{m}$ intervals and blue lines are coastlines. The " $A$ " marker identifies a valley with cold pool, "B" a hilltop above an inversion, "C" an urban heat island, and "D" coastal effects.

Sweden, Finland, Estonia, Latvia, and Lithuania. The Netatmo observations have helped us improve nowcast accuracy and keep short-range forecasts up to date; the products are reissued every hour. This article focuses on temperature forecasts, but we envision widespread integration of unconventional (citizen) observations in our automated forecast systems in the future.

\section{Citizen Observations}

Citizen observations do not follow World Meteorological Organization (WMO) rules for weather observations. For example, citizen stations can be located in backyards, on balconies, near walls, in direct sunlight, or even indoors. Measurements from poorly located instruments will not be representative of their area. Additionally, citizen weather stations typically lack metadata about their location and maintenance, so we cannot a priori determine the error characteristics of their measurements.

The strength of citizen observations is gained from their prevalence. Within Norway, the Netatmo network alone already outnumbers MET Norway's network of WMO-compliant stations by a factor of around 50. Coverage is most dense in cities along the coast; however, inland towns and mountainous areas with extensive cabin settlements are also well covered. We have focused our efforts on observations from Netatmo because of this coverage and because the programming interface allows access to data in near-real time.

Despite the uncertainty associated with individual citizen observations, citizen networks capture high-resolution weather phenomena, such as urban heat island effects, due to their sheer density. For example, in late March 2018, several key meteorological features in Oslo were represented by the Netatmo observations, including an overall 
temperature inversion, valley cold pools, an urban heat island, and a warmer coastline. These features, including the inversion, were not captured by MET Norway's operational NWP model. The case also showed some adjacent stations with contradictory temperatures-likely a result of poorly located stations. The general spatial distribution of temperature made physical sense, however.

\section{Spatial Quality Control}

Left unmodified, such noisy data can be problematic for operational systems. Unreliable data that are not quality controlled can lead to poor forecasts and reduced user confidence. Quality control (QC) is therefore essential when using citizen observations. When stations are properly located, regularly maintained, and calibrated, QC methods can focus on other sources of errors, such as faults in the sensors.

Sometimes, QC fails to identify the reason for systematic errors caused by ill-chosen locations of citizen weather stations. For example, a station placed too close to a building can exhibit diurnally varying biases that are difficult to detect by analyzing the time series of observations alone. As metadata are rarely available, a black list of stations known to be poorly located cannot be created. Manual, expert evaluation is not possible due to the large number of stations, and because operational
NWP requires quality-controlled observations to be available with little delay. The QC methods selected must therefore be fully automatic.

We have focused on using methods that exploit the spatial properties of the observing network instead of temporal properties of individual stations. The massive redundancy of stations means that in many cases, we have independent nearby observations available to help confirm or reject a particular observation.

The first spatial test we perform is the "buddy check," which compares an observed value against other (buddy) observations within a $3-\mathrm{km}$ radius and $30-\mathrm{m}$ elevation. The observation is removed if its deviation from the average is more than twice the standard deviation of the observations in the neighborhood. This removes unrealistic finescale variability in the observations.

Next, the spatial consistency test (SCT) is applied. For a given observation, the SCT computes an expected value and error variance based on the other observations. If the ratio of squared deviation to the cross-validation error variance is greater than 4 , the observation is removed. As most error sources for citizen observations contribute to a warm bias (e.g., direct sunlight or proximity to walls), we allow a less strict threshold (ratio of 8) for cold deviations from the expected. The process is repeated until no observations are removed.

\section{(a) Morning (05 UTC)}

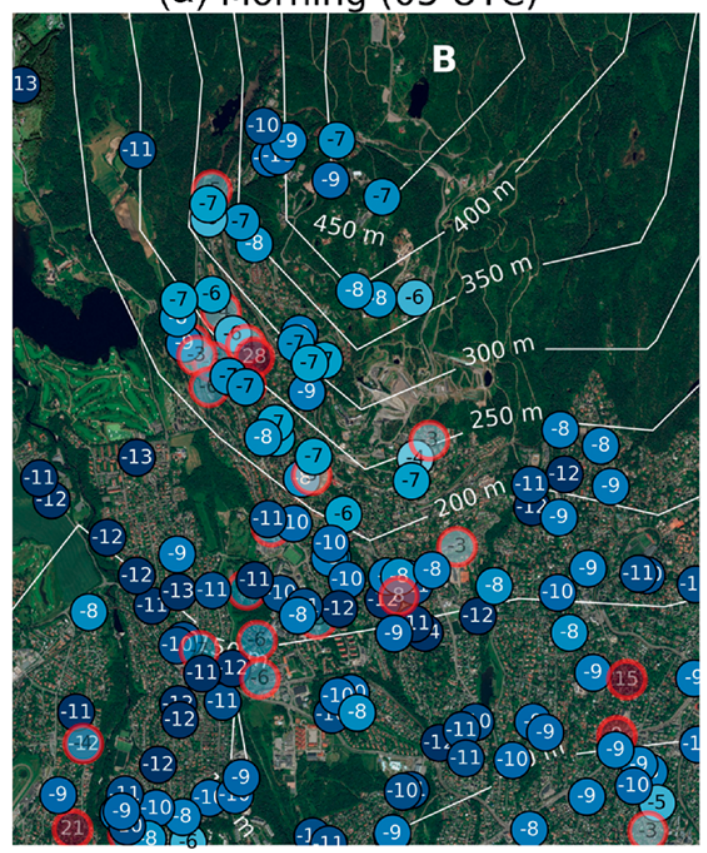

(b) Afternoon (13 UTC)

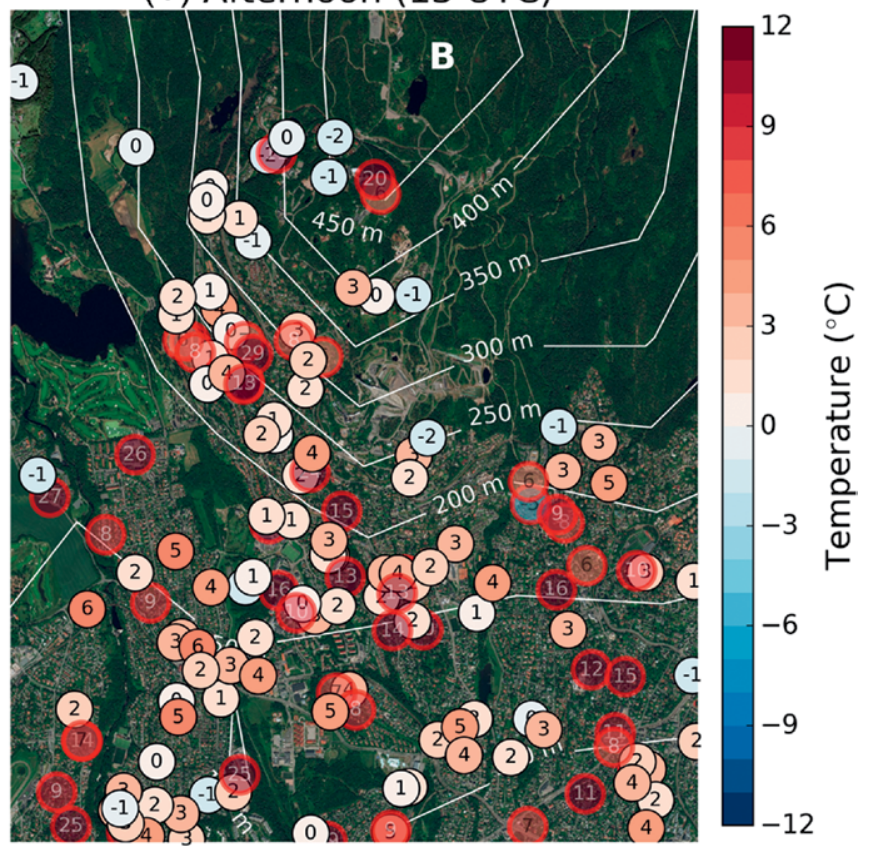




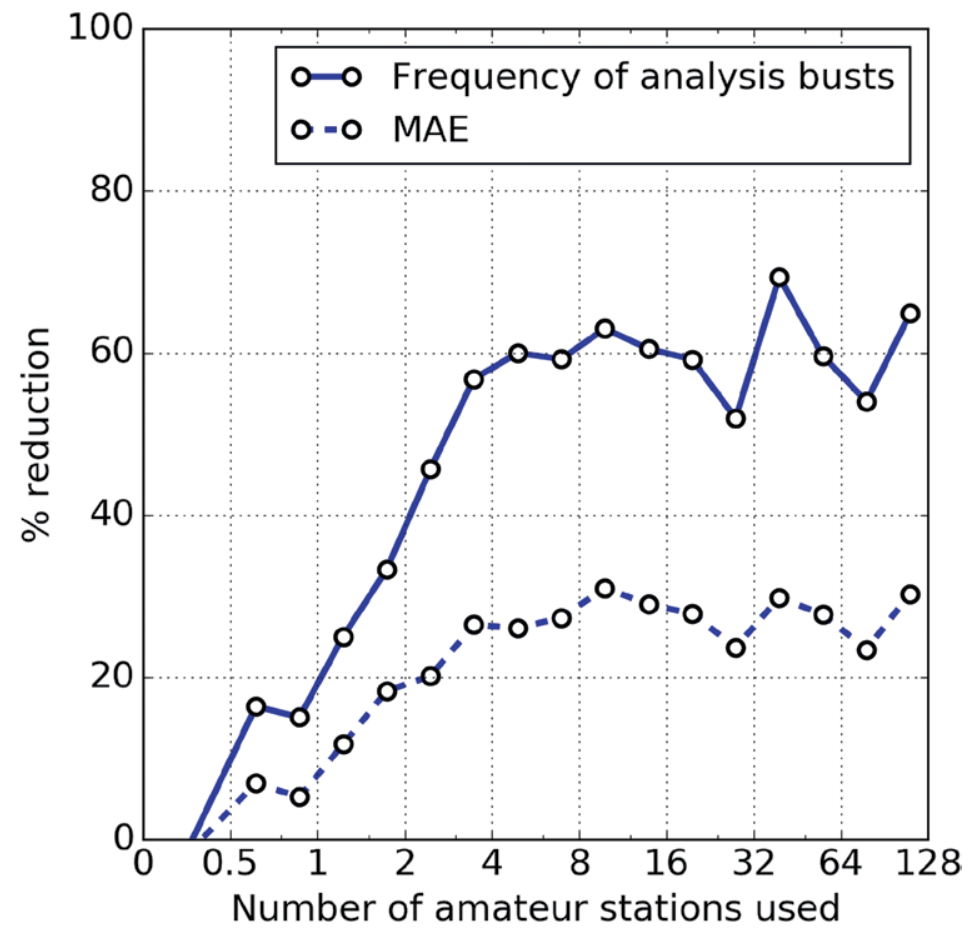

$\Delta$ Higher network densities result in greater analysis improvements. Improvements to the frequency of analysis busts (errors greater than $3^{\circ} \mathrm{C}$ ) and mean absolute error (MAE), however, saturate when more than $\mathbf{4}$ citizen stations are available. Analysis improvement is shown as the $\%$ reduction compared against the raw NWP. The network density at a particular point is computed by summing up the number of citizen stations that influence the point. Each observation is weighted by its distance to the gridpoint, so, for example, a sum of 4 could either represent a gridpoint where 4 citizen stations are exactly colocated with the gridpoint, 6 stations that are located $6 \mathrm{~km}$ away, or 8 stations that are located $12 \mathrm{~km}$ away.

The spatial approach implies that lone stations cannot be trusted since there is no independent information to validate them. Finally, we use an "isolation test" that removes stations that do not have at least 5 other stations within a $15-\mathrm{km}$ radius and $200-\mathrm{m}$ elevation difference.

The tests are performed independently each hour, which causes some stations to be removed at different times of the day or in different months of the year. This is especially true for stations that receive direct sunlight at specific sun directions. This time-independence allows observations from poorly located stations to contribute at times when their location does not lead to unrepresentative measurements.

On average, the tests remove $21 \%$ of observations. The spatial consistency test removes the most (16.3\% on average), followed by the isolation test (3.6\% on average), and finally the buddy check (1.5\% on average). The time-independent checks allow us to retain a much higher fraction of measurements than would otherwise be the case.

\section{Creating a Gridded Truth}

The remaining observations of temperature are now reasonably accurate. These are then used to create a gridded truth (often called an analysis) on a $1-\mathrm{km} \times 1-\mathrm{km}$ grid. The gridded truth gives the best estimate of the current conditions. In addition to the public forecasts, specialized users such as hydropower companies and flood prediction agencies use the gridded truth in their own models. The gridded truth also forms the basis for correcting our short-range weather forecasts.

We construct a gridded truth by combining NWP model output and available observations, where each data source is weighted by its certainty. The product is updated every hour with the latest observations and the most recent NWP run. The uncertainty of observations is generally much lower than for the NWP output. In areas with a high density of observations, the resulting analysis is therefore nearly a pure observation product, whereas in observation-sparse areas, the analysis is close to the raw NWP output.

To combine the two data sources, we use a technique frequently used in data assimilation-though in this situation we are interpolating the observations to create a gridded truth as opposed to initializing a model.

\section{Adjusting Short-Term Forecasts}

The next step is to correct the short-range forecasts, which currently extend $60 \mathrm{~h}$ into the future. Users expect a seamless transition from the current conditions to the short-range forecast, and consequently there can be no unrealistic jumps in temperature. The short-range forecasts must therefore be updated simultaneously with the current conditions at every hour and postprocessed on the same $1-\mathrm{km} \times 1-\mathrm{km}$ grid.

The need to postprocess gridded NWP forecasts is a challenge faced by most forecast 
BAMS: What would you like readers to learn from this article?

Thomas Nipen: I hope the readers will take home the message that citizen weather observations provide valuable data for improving operational weather forecasts. The key is to use suitable methods that handle the unique characteristics of the data source.

BAMS: What got you initially interested in meteorology or in the related field you are in?

TN: To this day, I still remember turning the page of my high school math textbook to the chapter on statistics, which happened to have a weather analysis map as its title image. This picture instantly sparked my interest for meteorology and ultimately led me down the path of studying the subject at university.

BAMS: How did you become interested in the topic of this article?

TN: Working on developing weather forecasts for the general public exposes you to huge amounts of feedback. We have read countless stories, many of a very personal and detailed character, of plans that have been spoiled by incorrect forecasts. Specifically, temperature forecasts in wintertime generate a lot of complaints. This motivated us to look for new observation data sources with better spatial coverage to help us deliver better forecasts.

BAMS: What surprised you the most about the work you document in this article?

TN: I was surprised at how much interest the work generated among the general public when we launched our operational product. Many of our users want to contribute to making the forecasts better, and this has given them a more direct way of doing so. providers. Here, the complex problem of postprocessing gridded fields is split into two operations. First, the spatial problem of determining how biases vary in space is handled by the gridded truth, and second, the temporal problem of how biases vary across forecast lead times is handled grid point by grid point.

Our point here is not to present a perfect postprocessing method, but rather to illustrate how the gridded-truth framework allows a high-density network of citizen observations to be integrated into the postprocessing of short-range forecasts.

\section{Impact on Accuracy}

It is important to establish the impact of the citizen network on the quality of the gridded truths and forecasts. For the impact evaluation, we rerun our forecast system without including WMO stations. The Netatmo-based analyses and forecasts are subsequently verified against the nonassimilated WMO observations.

On average, citizen observations reduce the mean absolute error of the gridded truth by $33 \%$ (from $1.24^{\circ}$ to $0.84^{\circ} \mathrm{C}$ ). The improvement is larger during winter, likely due to the prevalence of inversions that the NWP model often fails to capture. When the gridded truth uses observations that have not been quality controlled, it is only marginally better than the NWP forecast, and is worse in daytime and during the summer. Thus, QC is essential for getting added value from citizen observations.

Many of our end users are not sensitive to small forecast errors. We therefore also evaluate the system by focusing on large errors, which we will call analysis/forecast busts and are defined as errors greater than $3^{\circ} \mathrm{C}$. The citizen network reduces the frequency of analysis busts by $68 \%$ (a reduction from $8.1 \%$ to $2.6 \%$ ), an even larger impact than for the mean absolute error.

Other evaluation metrics also consistently show that the quality of the forecasts is generally better when integrating additional data provided by citizen observations (i.e., when the station density is high).

\section{Conclusions}

We've presented a possible pathway for integrating a vast network of citizen observations into operations at a national weather forecast service. The number of citizen observations is growing rapidly, and we believe these data will play a key role in providing high-quality forecasts on a local scale. This will increase the value of the forecasts for a range of applications in diverse societal sectors like energy production and distribution, transportation, agriculture, health, and water management. 
Find out from the authoritative source th

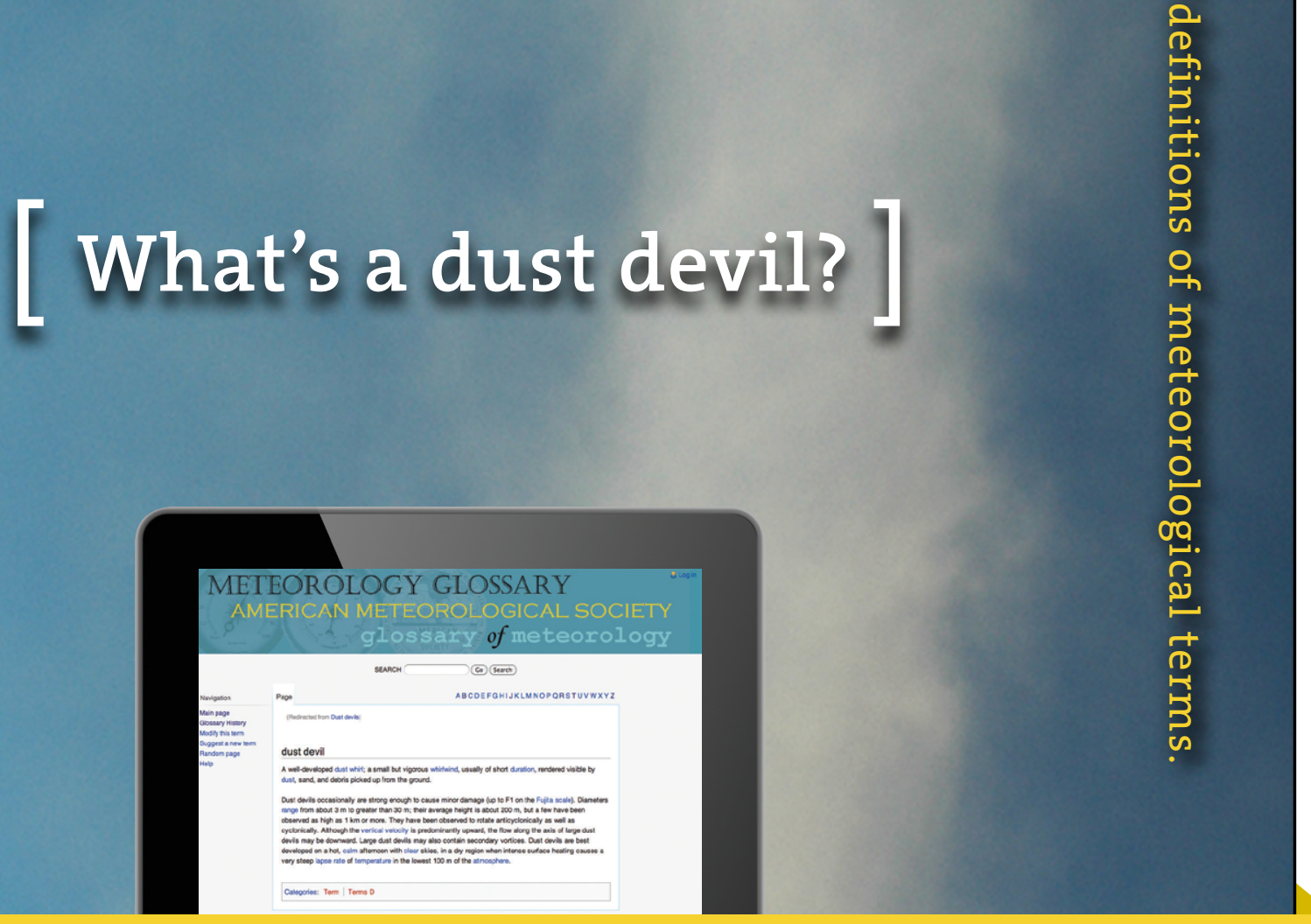

THE AMERICAN METEOROLOGICAL SOCIETY

\section{Online Glossary of Meteorology}

With over 12,000 meteorological terms, you'll be able to look up definitions online any time, any place, anywhere. http://glossary.ametsoc.org/wiki 